\begin{tabular}{|c|c|c|c|c|c|c|}
\hline \multirow{4}{*}{ Impact Factor: } & ISRA (India) & 3.117 & SIS (USA) & $=0.912$ & ICV (Poland) & $=6.630$ \\
\hline & ISI (Dubai, UAE & $=0.829$ & РИНЦ (Russia & $=0.156$ & PIF (India) & $=1.940$ \\
\hline & GIF (Australia) & $=0.564$ & ESJI (KZ) & $=5.015$ & IBI (India) & $=4.260$ \\
\hline & JIF & $=1.500$ & SJIF (Morocco & $=5.667$ & & \\
\hline
\end{tabular}

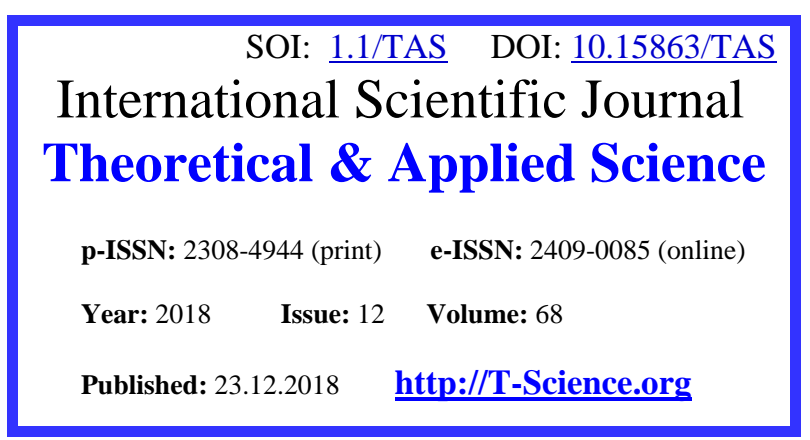

SECTION 4. Computer science, computer engineering and automation.
QR - Issue
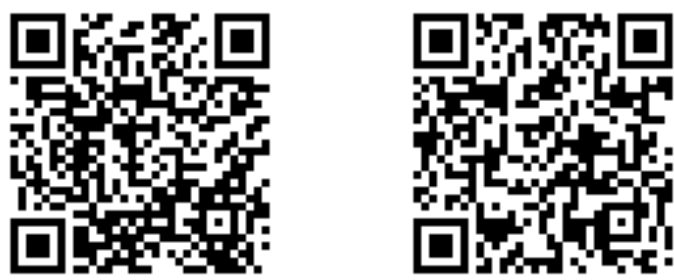

Victor Aleksandrovich Melent'ev

Philosophy Doctor, senior research associate Rzhanov Institute of Semiconductor Physics Siberian Branch of Russian Academy of Sciences (ISP SB RAS) melva@isp.nsc.ru

\title{
USE OF MELENTIEV'S GRAPH REPRESENTATION METHOD FOR DETECTION OF CLIQUES AND THE ANALYSIS OF TOPOLOGIES OF COMPUTING SYSTEMS
}

Abstract: The problem of comparison of the computing systems (CS) topologies is related with finding of maximum cliques in the CS graph. The solution of this task based on using of the representation of the graph offered by the Author is provided in this paper. Properties of projections of the complete graph and projections of cliques of general graph are formulated. The algorithm to identify the maximum clique of the given graph of the computing system and of its modifications corresponding to presented values of indices of reachability and faulttolerance is described and illustrated by the examples.

Key words: graph's projection, clique graph, reachability of vertexes, topological scalability and topological fault-tolerance of computing systems.

Language: Russian

Citation: Melent'ev, V. A. (2018). Use of Melentiev's graph representation method for detection of cliques and the analysis of topologies of computing systems. ISJ Theoretical \& Applied Science, 12 (68), 201-211.

Soi: http://s-o-i.org/1.1/TAS-12-68-28 Doi: crossef https://dx.doi.org/10.15863/TAS.2018.12.68.28

\section{ИСПОЛЬЗОВАНИЕ МЕТОДА МЕЛЕНТЬЕВА ПРЕДСТАВЛЕНИЯ ГРАФОВ ДЛЯ ВЫЯВЛЕНИЯ КЛИК И АНАЛИЗА ТОПОЛОГИЙ ВЫЧИСЛИТЕЛЬНЫХ СИСТЕМ}

Аннотация: Проблема сопоставления топологий вычислительных систем (ВС) связана с поиском наибольших клик в графе ВС. В работе представлено решение этой задачи, основанное на использовании предложенного Автором описания графа. Сформулированы свойства проекций полного графа и проекций клик произвольного графа. Описан и проиллюстрирован примерами алгоритм выявления наибольшей клики в заданном графе ВС и в его модификациях, соответствуюших заданным значениям показателей достижимости и отказоустойчивости.

Ключевые слова: проекция графа, клика графа, достижимость вершин, топологическая маситабируемость и топологическая отказоустойчивость вычислительных систем.

\section{Введение}

Разнообразие используемых в построении суперкомпьютеров топологий интерконнекта обусловило необходимость выбора и создания топологий на основе сопоставления ясных критериев, непосредственно демонстрирующих влияние топологии на достижение конечной цели создания суперкомпьютера - на повышение его вычислительной мощности до значений, требуемых для актуализированного решения определенного круга задач с присущими им объемами обрабатываемых данных и алгоритмами.
Традиционно используемые в сопоставлении системных топологий экспертные и метрические, а также такие сетевые характеристики как промежуточная центральность [1, с. 39], стабильность межузловых расстояний, вероятность потери связности, вероятность фрагментации, синхронизуемость [2, с. 1]) и пр. настолько неявны и субъективны, что влияние выбранной топологии на вычислительную мощность суперкомпьютера может быть количественно оценено только по результатам тестовых испытаний уже готового продукта. Установление же формальной обусловленности 


\begin{tabular}{|c|c|c|c|c|c|c|}
\hline \multirow{4}{*}{ Impact Factor: } & ISRA (India) & $=3.117$ & SIS (USA) & $=0.912$ & ICV (Poland) & $=6.630$ \\
\hline & ISI (Dubai, UAE & $=0.829$ & РИНЦ (Russia) & $=0.156$ & PIF (India) & $=1.940$ \\
\hline & GIF (Australia) & $=0.564$ & ESJI (KZ) & $=5.015$ & IBI (India) & $=4.260$ \\
\hline & JIF & $=1.500$ & SJIF (Morocco) & $=5.667$ & & \\
\hline
\end{tabular}

топологии потенцильному параллелизму позволяет оптимизировать выбор топологии еще на этапе проектирования суперкомпьютера и в соответствии $\mathrm{c}$ его проблемной ориентацией. Потенциал параллелизма рассматривается в [3, с. 138] в качестве обобщенной единицы и определяется предельным рангом параллельной задачи с лимитируемой достижимостью информационно-смежных ветвей. Введение понятия лимитируемой достижимости позволило абстрагироваться от используемых интерконнектом сетевых технологий, анализируя, таким образом, чисто топологические аспекты повышения потенциального параллелизма. Очевидно, что нижняя граница потенциального параллелизма определяется максимальным, обусловленным топологией, рангом информационно-полносвязной задачи, соответствующим мощности наибольшей максимальной по включению клики графа анализируемой ВС. Напомним в связи с этим, что кликой в графе называется любой полный подграф этого графа, т. е. любые две вершины клики смежны; максимальные по включению полные подграфы называются максимальными кликами графа. Число вершин в клике называют ее порядком, а порядок наибольшей из максимальных клик в графе - его плотностью [4, c. 1] или кликовым числом. Известно [5, с. 44-48], что задача о кликах графа $G$ в ее вычислительном варианте о поиске в заданном графе клики максимального размера трансформируется в задачу о множествах независимых вершин переходом к дополнительному графу $\bar{G}$ и относится, таким образом, к классу $N P$-полных задач. Понятно поэтому, что базирующаяся на поиске клик проблема оценки потенциального параллелизма вычислительных систем (BC) имеет этот же статус.

В работе представлен подход, основанный на методе проективного описания графов, называемом по имени Автора не только в русскоязычной - «V.A. Melentiev's Notation» [6, c. $83 ; 6$, с. 90], но и в англоязычной литературе «Melentiev's Graph Representation Method» [6, c. 1]; этот метод позволяет существенно снизить вычислительную сложность как выявления клик в графе ВС, так и сопоставления топологий интерконнекта с позиций обеспечиваемого ими потенциального параллелизма системы.

\section{Свойства проекций полного графа}

Используемый в данной работе метод [7] последовательно развивался Автором в работах [8-11]. Напомним лишь некоторые сведения об этом методе и об используемых в нем определениях.

Проекиия $P\left(v_{j}\right)$ графа $G(V, E)$ представляет собой многоуровневую конструкцию, на нулевом уровне которой расположена вершина $v_{j} \in V$, выбранная в качестве ракурсной; порожденное ею подмножество вершин первого уровня $V_{1 j} \subset V$ содержит все вершины ее окружения $\mathcal{N}\left(v_{j}\right) \backslash v_{j}$ без порождающей его вершины $v_{j}$, а $i$-й уровень $(i \geq$ 1) представляет собой совокупность подмножеств вершин, каждое из которых порождено вершиной $(i-1)$-го уровня и является окружением этой вершины без вершин, предшествующих ей в данной проекции. [12; с. 246]

Проекция $P\left(v_{j}\right)$ графа $G(V, E)$ является полной, если ею определены все вершины и все ребра (отношения смежности) этого графа [12; с. 247].

Если в проекции графа одна и та же вершина встречается $m$ раз, то ее экземпляр, расположенный первым слева на низшем уровне проекции, считаем оригинальньлм. Остальные $(m-1)$ ее копии называем реплицированными, или репликами (replica). [13, с. 22]

Граф называют полным, если любая пара вершин полного графа смежна. Вытекающее из этого определения основное свойство проекций полного графа состоит в том, что порожденное любой вершиной 1-го уровня подмножество вершин 2-го уровня любой проекции, включает в себя все вершины этого же уровня (за исключением порождающей) и, таким образом, любая 2-уровневая проекция полного графа является полной.

Тогда для выявления клик в графе, содержащих в составе некоторую вершину, достаточно построить 2-уровневую его проекцию с ракурсом из этой вершины и удалить из каждого порожденного на 2-м уровне подмножеств те вершины, которые отсутствуют на 1-м уровне. Продемонстрируем это на примере простого графа, изображенного на рис. 1 вместе с его 2уровневыми проекциями: 


\begin{tabular}{|c|c|c|c|c|c|c|}
\hline \multirow{4}{*}{ Impact Factor: } & ISRA (India) & $=3.117$ & SIS (USA) & $=0.912$ & ICV (Poland) & $=6.630$ \\
\hline & ISI (Dubai, UAE & $=0.829$ & РИНЦ (Russia) & $=0.156$ & PIF (India) & $=1.940$ \\
\hline & GIF (Australia) & $=0.564$ & ESJI (KZ) & $=5.015$ & IBI (India) & $=4.260$ \\
\hline & JIF & $=1.500$ & SJIF (Morocco) & $=5.667$ & & \\
\hline
\end{tabular}

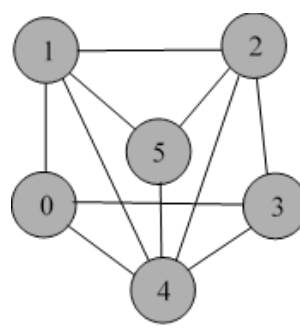

$$
\begin{aligned}
& P(0)=0^{\left(1^{(2,4,5)}, 3^{(2,4)}, 4^{(1,2,3,5)}\right),} \\
& P(1)=1^{\left(0^{(3,4)}, 2^{(3,4,5)}, 4^{(0,2,3,5)}, 5^{(2,4)}\right),} \\
& P(2)=2^{\left(1^{(0,4,5)}, 3^{(0,4)}, 4^{(0,1,3,5)}, 5^{(1,4)}\right)}, \\
& P(3)=3^{\left(0^{(2,4,5)}, 2^{(1,4)}, 4^{(0,1,2,5)}\right)}, \\
& P(4)=4^{\left(0^{(1,3)}, 1^{(0,2,5)}, 2^{(1,3,5)}, 3^{(0,2)}, 5^{(1,2)}\right),} \\
& P(5)=5^{\left(1^{(0,2,4)}, 2^{(1,3,4)}, 4^{(0,1,2,3)}\right) .}
\end{aligned}
$$

Рисунок 1 - Граф 1 и его проекции

Далее используем следующие обозначения: $P\left(v_{j}\right)$ - проекция рассматриваемого графа $G(V, E)$ с ракурсной вершиной $v_{j} \in V ;\left\{P\left(v_{j}\right)\right\}$ и $\left|P\left(v_{j}\right)\right|-$ множество и число таких проекций, соответственно.

$V_{1}\left(v_{j}\right)$ и $V_{2}\left(v_{j}\right)$ - множества вершин 1-го и 2го уровней в проекции $P\left(v_{j}\right) ; \quad V_{1}\left(v_{j}\right)$ есть подмножество вершин, порожденное ракурсной вершиной $v_{j}: V_{1}\left(v_{j}\right)=\left\{u_{i} \mid u_{i} \in \mathcal{N}\left(v_{j}\right)\right\}$, а $V_{2}\left(v_{j}\right)$ множество, объединяющее подмножества вершин $U\left(u_{i}\right)$, порожденные вершинами 1-го уровня: $V_{2}\left(v_{j}\right)=\bigcup\left\{U_{i} \mid U_{i}=\left\{\mathcal{N}\left(u_{i}\right) \backslash v_{j}\right\}\right\}$.

Понятно, что в полном графе $V_{1}\left(v_{j}\right)=V_{2}\left(v_{j}\right)$ и любое подмножество $U_{i}$ вершин, порожденное на втором уровне любой проекции, содержит все вершины $V$ такого графа без ракурсной и без порождающей это подмножество $U_{i}$ вершины 1-го уровня: $U_{i}=V \backslash v_{j} \backslash u_{i}$. Соответственно, в полном графе равны и мощности этих подмножеств: $\forall u_{i} \in$ $V_{1} m\left(u_{i}\right)=\left|U_{i}\right|=|V|-2=m, \quad n_{1}=\left|V_{1}\right|=m+$ $1=|V|-1, m=n_{1}-1$, или $V=n_{1}+1=m+2$.

Естественно, все вышесказанное справедливо не только для полного графа, но и для клик любого графа, поэтому для выявления клик достаточно построить 2-уровневые проекции исследуемого графа и исключить из этих проекций вершины, не соответствующие свойствам проекций полного подграфа.

Таким образом, если на внешнем уровне какой-либо из множества $\left\{P\left(v_{j}\right)\right\}$ 2-уровневых проекций рассматриваемого графа существует максимальное по числу вершин подмножество $M\left(v_{j}\right)$ мощностью $m\left(v_{j}\right)=\left|M\left(v_{j}\right)\right|=\max _{1 \leq i \leq n_{1}}\left\{\left|U_{i}\right|\right\}$, то для существования клики порядка $m\left(v_{j}\right)+2$ с входящей в ее состав ракурсной вершиной рассматриваемой проекции на уровне 2 должно находиться не менее, чем $m\left(v_{j}\right)+1$ подмножеств мощности $m\left(v_{j}\right)$, - это же относится и к числу порождающих эти подмножества вершин 1-го уровня.

Продемонстрируем это на конкретном примере приведенных на рис. 1 графа и его проекций. Первый уровень проекции $P(0)$ содержит 3 вершины: $V_{1}(0)=\{1,3,4\}$. Это означает, что порядок максимальной клики $K(G)$, содержащей в своем составе вершину 0 , не может быть большим четырех $(|K(G)| \leq 3+1)$ Учитывая, что подмножества вершин 2-го уровня не могут включать в себя вершины, не входящие в $V_{1}(0)$, из проекции таковые исключаем, оставив в подмножествах 2-го уровня только вершины 1-го уровня:

$$
P(0)=0^{\left(1^{(4)}, 3^{(4)}, 4^{(1,3)}\right)} .
$$

Максимальное подмножество 2-го уровня $M(0)=(1,3)$ единственно и содержит всего две $(m(0)=|M(0)|=2)$ вершины, и если бы такое подмножество было не одно, а столько же, сколько и вершин 1-го уровня $-n_{1}(0)=3$, то мы могли бы рассмотреть возможность существования клики порядка 4. Однако, подмножество мощности $m(0)=2$ всего одно, поэтому максимальная клика не может иметь здесь порядок, больший трех. Выявить такие клики (их вершины выделены здесь жирным шрифтом) не представляет сложности:

$$
\begin{aligned}
& P(0)=\mathbf{0}^{\left(1^{(4)}, 3^{(4)}, \mathbf{4}^{(1,3)}\right)}, \text { клика }(0,1,4), \\
& P(0)=\mathbf{0}^{\left(1^{(4)}, \mathbf{3}^{(4)}, \mathbf{4}^{(1,3)}\right)}, \text { клика }(0,3,4) .
\end{aligned}
$$

Но так как нас интересует не любая, а наибольшая максимальная клика, то те же действия, что и для $P(0)$ произведем для остальных пяти проекций графа:

$$
\begin{gathered}
P(1)=1^{\left(0^{(4)}, 2^{(4,5)}, 4^{(0,2,5)}, 5^{(2,4)}\right),} \\
P(2)=2^{\left(1^{(4,5)}, 3^{(4)}, 4^{(1,3,5)}, 5^{(1,4)}\right)}, \\
P(3)=3^{\left(0^{(2,4)}, 2^{(4)}, 4^{(0,2)}\right)}, \\
P(4)=4^{\left(0^{(1,3)}, 1^{(0,2,5)}, 2^{(1,3,5)}, 3^{(0,2)}, 5^{(1,2)}\right),} \\
P(5)=5^{\left(1^{(2,4)}, 2^{(1,4)}, 4^{(1,2)}\right)} .
\end{gathered}
$$

Как видим, проекция $P(3)$ также, как и $P(0)$ не соответствует требованию большего, чем 3 , порядка клики $(m(3)>2), \quad$ поэтому рассматриваем и корректируем остальные 4 проекции, исключив из подмножеств этих проекций вершины 0 и 3 (выше мы выяснили, что эти вершины не могут быть в составе клик с порядком, большим трех):

$$
\begin{aligned}
& P(1)=1^{\left(2^{(4,5)}, 4^{(2,5)}, 5^{(2,4)}\right),} \\
& P(2)=2^{\left(1^{(4,5)}, 4^{(1,5)}, 5^{(1,4)}\right),}
\end{aligned}
$$




\begin{tabular}{|c|c|c|c|c|c|c|}
\hline \multirow{4}{*}{ Impact Factor: } & ISRA (India) & $=3.117$ & SIS (USA) & $=0.912$ & ICV (Poland) & $=6.630$ \\
\hline & ISI (Dubai, UAE & $=0.829$ & РИНЦ (Russia) & $=0.156$ & PIF (India) & $=1.940$ \\
\hline & GIF (Australia) & $=0.564$ & ESJI (KZ) & $=\mathbf{5 . 0 1 5}$ & IBI (India) & $=4.260$ \\
\hline & JIF & $=1.500$ & SJIF (Morocco) & $=5.667$ & & \\
\hline
\end{tabular}

$$
\begin{aligned}
& P(4)=4^{\left(1^{(2.5)}, 2^{(1.5)}, 5^{(1,2)}\right)}, \\
& P(5)=5^{\left(1^{(2,4)}, 2^{(1,4)}, 4^{(1,2)}\right)} .
\end{aligned}
$$

В каждой из полученных здесь проекций подмножества, образуемые любой вершиной 1-го уровня и порожденными ею вершинами 2-го уровня, совпадают, и наибольшая клика в рассматриваемом графе состоит из четырех вершин $(1,2,4,5)$. Далее дадим описание соответствующего алгоритма.

\section{Алгоритм выявления наибольшей клики}

Итак, для выявления наибольшей клики в графе $G(V, E)$ производим следующую последовательность действий:

1. Строим множество $\left\{P\left(v_{j}\right), v_{j} \in V\right\}$ из $n=$ $|V|$ двухуровневых проекций. В каждой из этих проекций $\quad V_{1}\left(v_{j}\right)=\left\{u_{i} \mid u_{i} \in \mathcal{N}\left(v_{j}\right)\right\}, \quad U_{i}=$ $\left\{\mathcal{N}\left(u_{i}\right) \backslash v_{j}\right\} ;$

2. Вторые уровни проекций этого множества $\left\{P\left(v_{j}\right), v_{j} \in V\right\}$ корректируем, оставив в каждом из порожденных на них подмножеств только реплики вершин 1-го уровня и получив, таким образом, $\quad$ множество $\left\{P^{\prime}\left(v_{j}\right), v_{j} \in V\right\}$ проекций $P^{\prime}\left(v_{j}\right)$, в которых множество вершин 1-го уровня остается прежним $V_{1}{ }^{\prime}\left(v_{j}\right)=V_{1}\left(v_{j}\right)=\left\{u_{i} \mid u_{i} \in\right.$ $\left.\mathcal{N}\left(v_{j}\right)\right\}, \quad$ а $\quad U_{i}{ }^{\prime}=U_{i} \cap V_{1}\left(v_{j}\right)$. Соответственно, $V_{2}^{\prime}\left(v_{j}\right):=V_{2}\left(v_{j}\right) \cap V_{1}\left(v_{j}\right)$.

3. В каждой проекции $P^{\prime}\left(v_{j}\right)$ определяем максимальную мощность $m\left(v_{j}\right)$ подмножеств 2-го уровня и выбираем наибольшее из них значение $m:=\max _{1 \leq j \leq n} m\left(v_{j}\right)$.

При $m=0$, т. е. все подмножества 2-го уровня каждой проекции из $\left\{P^{\prime}\left(v_{i}\right)\right\}$ пусты, порядок наибольшей клики равен двум, и кликой является любая пара смежных в рассматриваемом графе вершин, и в этом случае Алгоритм может быть завершен. При $m>0$ переходим к выполнению п. 4;

4. Формируем множество проекций $\left\{P^{\prime \prime}\left(v_{i}\right)\right\}$, удаляя из проекций $\left\{P^{\prime}\left(v_{i}\right)\right\}$ подмножества 2-го уровня, мощности которых меньше $m$, удаляем из $\left\{P^{\prime \prime}\left(v_{i}\right)\right\}$ вершины 1-го уровня, порождающие эти подмножества.

В проекциях из получившегося таким образом множества $\left\{P^{\prime \prime}\left(v_{i}\right)\right\}$ оставляем только ракурсные вершины входящих в $\left\{P^{\prime \prime}\left(v_{i}\right)\right\}$ проекций и корректируем текущее значение $m$.

Отметим, что в принципе, алгоритм может также использоваться для получения всех клик заданного порядка $k$, для этого переменной $m$ следует присвоить значение $m:=k-2$.

Продемонстрируем формальное применение алгоритма на том же примере графа 1 (рис.1):
1. Множество $\left\{P\left(v_{j}\right), v_{j} \in V\right\}$ всех $n=|V|=$ 6 двухуровневых проекций рассматриваемого графа уже приведено на рис. 1.

2. Корректируем вторые уровни оставив на них только реплики вершин 1-го уровня:

$$
\begin{aligned}
& P^{\prime}(0)=0^{\left(1^{(4)}, 3^{(4)}, 4^{(1.3)}\right)} \text {, } \\
& P^{\prime}(1)=1^{\left(0^{(4)}, 2^{(4,5)}, 4^{(0,2.3)}, 5^{(2,4)}\right)}, \\
& P^{\prime}(2)=2^{\left(1^{(4.5)}, 3^{(4)}, 4^{(1,3.5)}, 5^{(1.4)}\right)}, \\
& P^{\prime}(3)=3^{\left(0^{(2,4)}, 2^{(4)}, 4^{(0.2)}\right)} \\
& P^{\prime}(4)=4^{\left(0^{(1.3)}, 1^{(0,2.5)}, 2^{(1.3 .3)}, 3^{(0.2)}, 5^{(1.2)}\right)} \text {, } \\
& P^{\prime}(5)=5^{\left(1^{(2,4)}, 2^{(1.4)}, 4^{(1.2)}\right)} \text {. }
\end{aligned}
$$

3. $m=\max _{0 \leq j \leq 5} m\left(v_{j}\right)=3$.

4. Из множества $\left\{P^{\prime}\left(v_{j}\right)\right\}$ исключаем подмножества с $m\left(v_{j}\right)<3$ и оставляем в этих подмножествах только реплики вершин 1-го уровня. Получаем проекции $P^{\prime \prime}\left(v_{j}\right)$ множества $\left\{P^{\prime \prime}\left(v_{j}\right)\right\}$

$$
\begin{aligned}
P^{\prime \prime}(1) & =1^{\left(4^{0}\right)}, \\
P^{\prime \prime}(2) & =2^{\left(4^{0}\right)}, \\
P^{\prime \prime}(4) & =4^{\left(1^{(2)}, 2^{(1)}\right),},
\end{aligned}
$$

Как видим, проекция $P^{\prime \prime}(4)$ задает 3-клику $K_{3}(G)=(1,2,4)$, и это не соответствует нашим ожиданиям получения клики из пяти (при $m=3$ ) вершин.

5. Так как полученный в п. 4 результат хуже ожидаемого $\quad\left(\left(\left|K_{3}(G)\right|=3\right)<(m+2=5)\right)$, уменьшаем разрешенное значение максимальной мощности подмножеств 2-го уровня до следующего по убыванию значения $m:=2$ и возвращаемся к п. 4:

4.1. Исключив из множества $\left\{P^{\prime}\left(v_{j}\right)\right\}$ проекции с $m\left(v_{j}\right)<2$, получим $\left\{P^{\prime \prime}\left(v_{j}\right)\right\}$ :

$$
\begin{aligned}
& P^{\prime \prime}(1)=1^{\left(2^{(4,5)} 4^{(2,5)}, 5^{(2,4)}\right)} \text {, } \\
& P^{\prime \prime}(2)=2^{\left(1^{(4,5)}, 4^{(1,5)}, 5^{(1,4)}\right)}, \\
& P^{\prime \prime}(4)=4^{\left(0^{(1.3)}, 1^{(0.2 .5)}, 2^{(1.3 .3)}, 3^{(0.2)}, 5^{(1.2)}\right)}, \\
& P^{\prime \prime}(5)=5^{\left(1^{(2,4)}, 2^{(1,4)}, 4^{(1,2)}\right)} \text {. }
\end{aligned}
$$

Из этих проекций удаляем вершины 0,3 (оставляем в проекциях только вершины 1, 2, 4, 5 - ракурсные вершины проекций из $\left.\left\{P^{\prime \prime}\left(v_{j}\right)\right\}\right)$ :

$$
\begin{aligned}
& P^{\prime \prime}(1)=1^{\left.\left(2^{(4.5)}\right) 4^{(2.5)}, 5^{(2.4)}\right)}, \\
& P^{\prime \prime}(2)=2^{\left(1^{(4.5)}, 4^{(1.5)}, 5^{(1.4)}\right)}, \\
& P^{\prime \prime}(4)=4^{\left(1^{(2,5)}, 2^{(1.5)}, 5^{(1.2)}\right)}, \\
& P^{\prime \prime}(5)=5^{\left(1^{(1,2)}, 2^{(1.4)}, 4^{(1.2)}\right)},
\end{aligned}
$$

Таким образом, для $m=2$ получили: $\left|P^{\prime \prime}\left(v_{j}\right)\right|=m+2=4, \quad n_{1}=m+1=3, \quad V(1)=$ $V(2)=V(3)=V(4)=(1,2,4,5) \quad$ и $\quad K_{\max }(G)=$ $K_{4}(G)=(1,2,4,5)$.

\section{Топологическая масштабируемость ВС}

Понятие топологической масштабируемости параллельных систем и задач неразрывно связано с проблемой оптимального вложения задачи в систему. 


\begin{tabular}{llllll} 
& ISRA (India) $=\mathbf{3 . 1 1 7}$ & SIS (USA) $=\mathbf{0 . 9 1 2}$ & ICV (Poland) & $\mathbf{= 6 . 6 3 0}$ \\
Impact Factor: & ISI (Dubai, UAE) $=\mathbf{0 . 8 2 9}$ & PUHL (Russia) $=\mathbf{0 . 1 5 6}$ & PIF (India) & $=\mathbf{1 . 9 4 0}$ \\
& GIF (Australia) $=\mathbf{0 . 5 6 4}$ & ESJI (KZ) & $\mathbf{5 . 0 1 5}$ & IBI (India) & $=\mathbf{4 . 2 6 0}$ \\
& JIF & $\mathbf{1 . 5 0 0}$ & SJIF (Morocco) $=\mathbf{5 . 6 6 7}$ & & \\
\hline
\end{tabular}

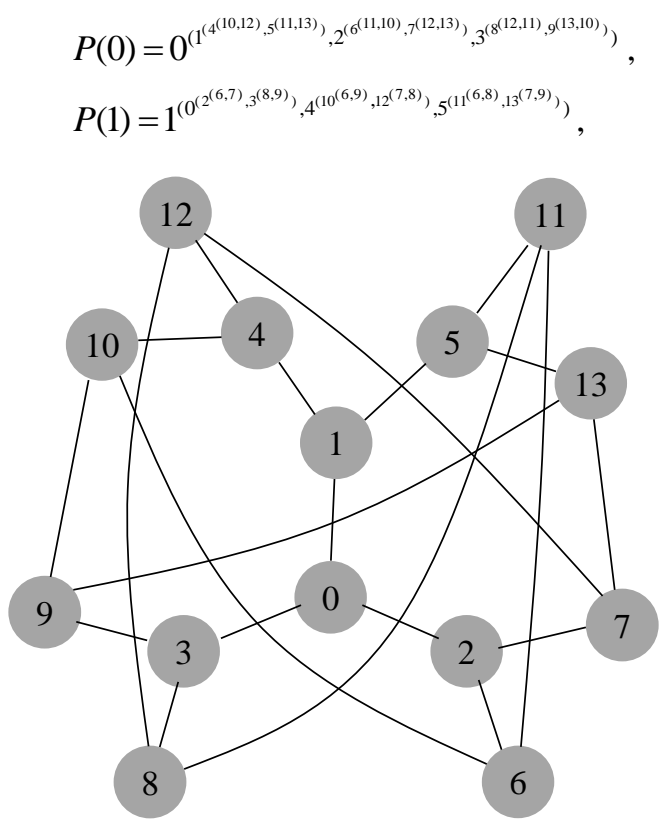

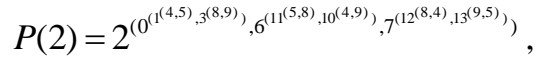

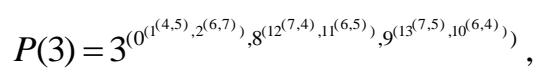

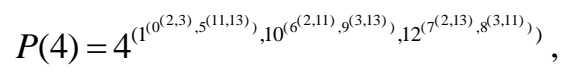

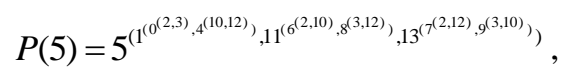

$$
\begin{aligned}
& P(6)=6^{\left(2^{\left(0^{(1,3)}, 7^{(12,13)}\right.}, 11^{\left(5^{(1,13)}, 8^{(3.12)}\right.}, 10^{\left(4^{(1,12)}, 9^{(3,13)}\right)},\right.} \text {, }
\end{aligned}
$$

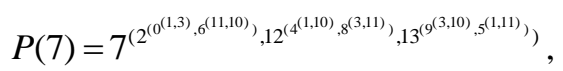

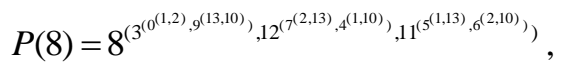

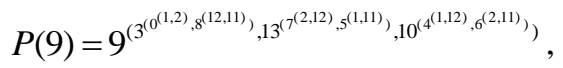

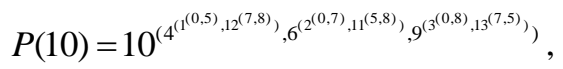

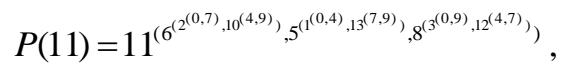

$$
\begin{aligned}
& P(12)=12^{\left(7^{\left(2^{(0.6)}, 13^{(9.5)}\right.}, 8^{\left.\left(3^{(0.9)}\right) 11^{(5.6)}\right)}, 4^{\left(4^{(0.5)} .10^{(6.9)}\right)}\right)},
\end{aligned}
$$

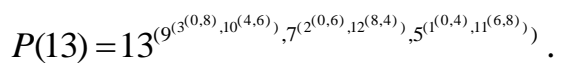

Рисунок 2. Граф 2 и множество $\left\{P\left(v_{j}\right)\right\}$ его полных 3-уровневых проекций.

Это понятие впервые введено в [3, с. 132], где представлено решение проблемы вложения в постановке, основанной на замене отношений смежности вершин графа ВС отношениями их достижимости; при этом вершины считаются достижимыми, если расстояние между ними не превышает порогового для рассматриваемой задачи значения достижимости $\partial[14$, c. $213 ; 15$, с. 123].

Максимальное по включению подмножество вершин, связанных между собой отношением Ә-достижимости, порождает клику $K\left(G_{\partial}\right)$ соответствующего графа $G_{\partial}$, иначе $-\partial$-клику $K_{\partial}(G)$ графа $G$ системы. Подобно плотности $\varphi(G)$ графа $G$, определяемой порядком его наибольшей клики $[16$, c. 21$]$, порядок наибольшей из максимальных $\partial$-клик графа $G(V, E)$ назван плотностью $\varphi\left(G_{\partial}\right)$ графа $\partial$-достижимости, или $\partial$-плотностью $\varphi_{\partial}(G)$ графа $G(V, E): \quad \varphi\left(G_{\partial}\right) \equiv$ $\varphi_{\partial}(G)$.

Порядок $n_{\partial}$ наибольшей в графе ВС $\partial$-клики $K_{\partial}(G)$, все вершины которой взаимно $\partial$-достижимы по определению, есть $\partial$-плотность $\varphi_{\partial}(G) \equiv n\left(K_{\partial}(G)\right.$ этого графа, он ограничивает сверху параллелизм (число ветвей) для информационно-полносвязных задач. Поэтому функция масштабируемости $\mathrm{BC} \mu_{\partial}(G)$ определена в [3, с. 138] для присущих графу $G$ достижимостей $1 \leq \partial \leq d(G)$ от единичного значения до диаметра $d(G)$, и измеряется нормированной масштабируемым порядком $n(G)$ плотностью $\varphi_{\partial}(G): \mu_{\partial}(G)=\varphi_{\partial}(G) / n(G)$.

Итак, чтобы оценить топологическую масштабируемость системы, необходимо определить соответствующую заданному значению достижимости $\partial$ плотность $\varphi_{\partial}(G)$ графа $G$, что мы и продемонстрируем далее на примере, более сложном, чем рассмотренный ранее граф 1 , на графе 2, представленном ниже на рис. 2 с множеством его полных проекций.

1. Для выявления максимальных клик при $\partial=1$ преобразуем проекции $\left\{P\left(v_{j}\right)\right\}$ в 2уровневые:

$$
\begin{aligned}
& P(0)=0^{\left(1^{(4,5)}, 2^{(6,7)}, 3^{(8,9)}\right)}, \quad P(1)=1^{\left(0^{(2,3)}, 4^{(10,12)}, 5^{(11,13)}\right)}, \\
& P(2)=2^{\left(0^{(1,3)}, 6^{(11,10)}, 7^{(12,13)}\right)}, \quad P(3)=3^{\left(0^{(1,2)}, 8^{(12,11)}, 9^{(13,10)}\right)^{\prime}}, \\
& P(4)=4^{\left(1^{(0.5)}, 10^{(6.9)}, 12^{(7.8)}\right)}, \quad P(5)=5^{\left(1^{(0.4)}, 11^{(6.8)}, 13^{(7.9)}\right)}, \\
& P(6)=6^{\left(2^{(0.7)}, 11^{(5.8)}, 10^{(4.9)}\right)}, \quad P(7)=7^{\left(2^{(0.6)}, 12^{(4.8)}, 13^{(9.5)}\right)}, \\
& P(8)=8^{\left(3^{(0.9)}, 12^{(7,4)}, 11^{(5,6)}\right),}, \quad P(9)=9^{\left(3^{(0.8)}, 11^{(7.5)}, 10^{(4,6)},\right.}, \\
& P(10)=10^{\left(4^{(1,12)}, 6^{(2,11)}, 9^{(3,13)}\right)}, \quad P(11)=11^{\left(6^{(2,10)}, 5^{(1,13)}, 8^{(3,12)}\right)} \\
& \left.P(12)=12^{\left(7^{(2.13)}, 8^{(3.11)}, 4^{(1.10)}\right)}, \quad P(13)=13^{\left(9^{(3.10)}, 7^{(2,12)}, 5^{(1.11)}\right.}\right)^{\prime} \text {. }
\end{aligned}
$$

2. Как видим, вершины 2-го уровня любой из полученных в п. 1 проекций не имеют пересечений с вершинами 1-го уровня, поэтому все подмножества порожденных на вторых уровнях вершин - пусты:

$$
\begin{array}{ll}
P^{\prime}(0)=0^{\left(1^{0}, 2^{0}, 3^{0}\right)}, & P^{\prime}(1)=1^{\left(0^{0}, 4^{0}, 5^{0}\right)}, \\
P^{\prime}(2)=2^{\left(0^{0}, 6^{0}, 7^{0}\right)}, & P^{\prime}(3)=3^{\left(0^{0}, 8^{0}, 9^{0}\right)}, \\
P^{\prime}(4)=4^{\left(1^{0}, 10^{0}, 12^{0}\right)}, & P^{\prime}(5)=5^{\left(1^{0}, 11^{0}, 10^{0}\right)}, \\
P^{\prime}(6)=6^{\left(2^{0}, 11^{0}, 10^{0}\right)}, & P^{\prime}(7)=7^{\left(2^{0}, 12^{0}, 13^{0}\right)}, \\
P^{\prime}(8)=8^{\left(3^{0}, 12^{0}, 11^{0}\right)}, & P^{\prime}(9)=9^{\left(3^{0}, 13^{0}, 10^{0}\right),} \\
P^{\prime}(10)=10^{\left(4^{0}, 6^{0}, 9^{0}\right)}, & P^{\prime}(11)=11^{\left(6^{0}, 5^{0}, 8^{0}\right)}, \\
P^{\prime}(12)=12^{\left(7^{0}, 8^{0}, 4^{0}\right)}, & P^{\prime}(13)=13^{\left(9^{0}, 7^{0}, 5^{0}\right)} .
\end{array}
$$

3. Получаем $m:=\max m_{2}\left(P_{2}\left(v_{i}\right)\right)=0$.

Таким образом, порядок наибольшей клики 


\begin{tabular}{|c|c|c|c|c|c|c|}
\hline \multirow{4}{*}{ Impact Factor: } & ISRA (India) & $=3.117$ & SIS (USA) & $=0.912$ & ICV (Poland) & $=6.630$ \\
\hline & ISI (Dubai, UAE & $=0.829$ & РИНЦ (Russia) & $=0.156$ & PIF (India) & $=1.940$ \\
\hline & GIF (Australia) & $=0.564$ & ESJI (KZ) & $=\mathbf{5 . 0 1 5}$ & IBI (India) & $=4.260$ \\
\hline & JIF & $=1.500$ & SJIF (Morocco) & $=5.667$ & & \\
\hline
\end{tabular}

$n\left(K_{\max }(G)=m+2=2, \varphi_{\partial=1}(G)=2\right.$ и кликами является любая пара смежных вершин нашего графа.

Соответственно, масштабируемость $\mu_{1}(G)$ рассматриваемого графа $G$ при $\partial=1$ :

$$
\mu_{1}(G)=\varphi_{1}(G) / n(G)=2 / 14=0,143 .
$$

Чтобы определить плотность и масштабируемость графа для достижимости $\partial>$ 1, модифицируем исходные проекции переместив в каждой проекции все вершины $V_{i}$ уровней $i(1<$ $i \leq \partial+1)$ на 1-й уровень: $V_{1}=\bigcup_{i=2}^{\partial+1} V_{i}$. Получив таким образом 1-уровневые проекции, фактически определяющие все отношения смежности в графе ?-достижимости $G_{\partial}$, надстраиваем эти проекции до 2-го уровня, заменяя вершины 1-го уровня их одноуровневыми проекциями. Понятно, что эти проекции полнотой не обладают, но, как мы указывали выше, для выявления клик достаточно всего двух уровней. Далее, для большей наглядности, мы сразу же упорядочиваем подмножества вершин каждого уровня по возрастанию их номеров.

Итак, для достижимости $\partial=2$ приведенные на рис. 2 проекции графа преобразуем вначале в 1уровневые проекции 2-достижимости:

$$
\begin{array}{ll}
P(0)=0^{(1,2,3,4,5,6,7,8,9)}, & P(1)=1^{(0,2,3,4,5,10,11,12,13)}, \\
P(2)=2^{(0,1,3,6,7,10,11,12,13)}, & P(3)=3^{(0,1,2,8,9,10,11,12,13)}, \\
P(4)=4^{(0,1,5,6,7,7,9,910,12)}, & P(5)=5^{(0,1,4,6,7,8,9,11,13)}, \\
P(6)=6^{(0,2,4,5,7,8,9,10,11)}, & P(7)=7^{(0,2,4,5,5,6,8,9,12,13)}, \\
P(8)=8^{(0,3,4,5,6,7,9,11,12)}, & P(9)=9^{(0,3,4,5,6,7,8,10,13),} \\
P(10)=10^{(1,2,3,4,6,9,11,12,13)}, & P(11)=11^{(1,2,3,5,6,8,10,12,13)}, \\
P(12)=12^{(1,2,3,4,7,7,10,11,13)}, & P(13)=13^{(1,2,3,5,5,7,9,10,11,12)} .
\end{array}
$$

Затем переходим к выполнению, собственно, Алгоритма:

1. Преобразуем эти проекции в 2-уровневые, заменяя вершины 1-го уровня их 1-уровневыми проекциями:

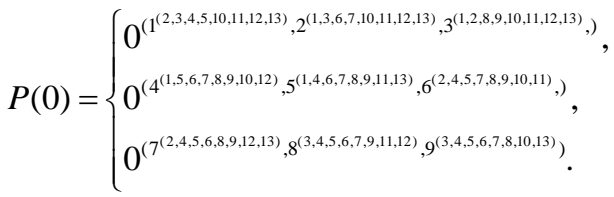

$$
\begin{aligned}
& P(1)=\left\{\begin{array}{l}
1^{\left(0^{(2,3,4,5,5,6,7,8,9)}, 2^{(0,3,6,6,7,10,11,12,13)}, 3^{(0,2,8,9,10,11,12,13)},\right)}, \\
1^{\left(4^{(0,5,6,6,7,8,9,10,12)}, 5^{(0,4,6,6,7,8,9,11,13)}, 10^{(2,3,4,4,6,9,11,12,13)},\right)}, \\
1^{\left(11^{(2,3,3,6,6,8,10,12,13)}, 12^{(2,3,4,4,7,8,10,11,13)}, 13^{(2,3,3,5,7,9,10,11,11,12)}\right)} .
\end{array}\right.
\end{aligned}
$$

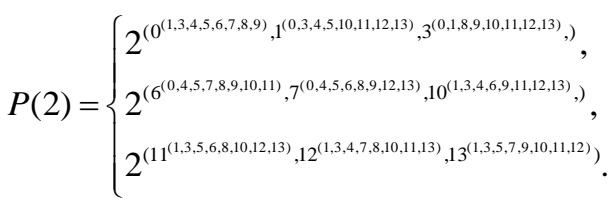

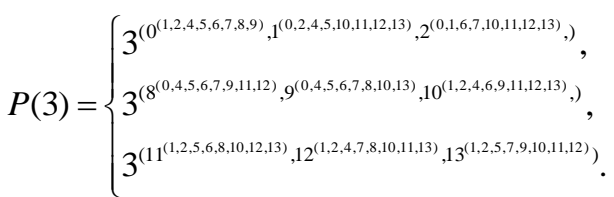

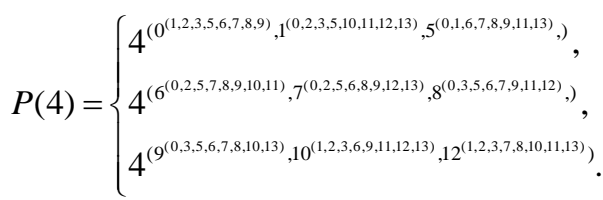

$P(5)=\left\{\begin{array}{l}5^{\left(0^{(1,2,3,3,4,6,7,8,9)}, 1^{(0,2,3,4,10,11,12,13)}, 4^{(0,1,6,7,7,8,9,10,12)},\right)}, \\ 5^{\left(6^{(0,2,4,4,7,8,9,10,11)}, 7^{(0,2,4,4,6,8,9,12,13)}, 8^{(0,3,4,4,6,7,9,11,12)},\right)}, \\ 5^{\left(9^{(0,3,4,6,6,7,8,10,13)}, 11^{(1,2,3,3,6,8,10,12,13)}, 13^{(1,2,3,3,7,9,10,11,12)}\right)} .\end{array}\right.$

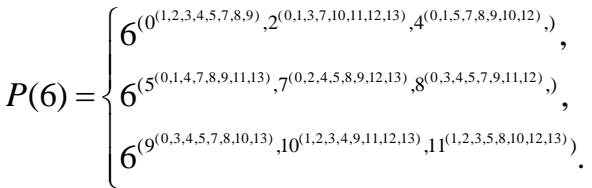

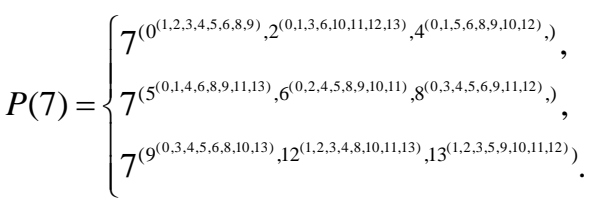

$P(8)=\left\{\begin{array}{l}8^{\left(0^{(1,2,3,3,4,5,6,7,9)}, 3^{(0,1,2,9,10,11,12,13)}, 4^{(0,1,5,6,6,7,9,10,12)},\right)}, \\ 8^{\left(5^{(0,1,1,4,6,7,9,11,13)}, 6^{(0,2,4,4,7,7,1,10,11)}, 7^{(0,2,4,4,6,6,9,12,13)},\right)}, \\ 8^{\left(9^{(0,3,4,4,5,6,7,10,13)}, 11^{(1,2,2,3,5,6,10,12,12,13)}, 12^{(1,2,2,3,4,7,7,10,11,13)}\right)} .\end{array}\right.$

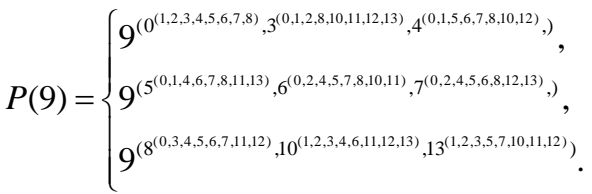

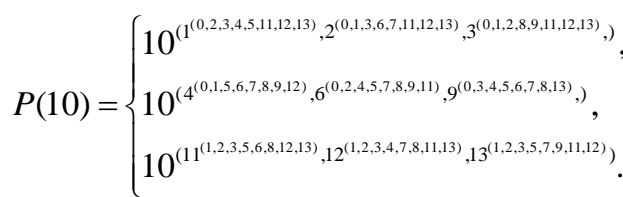

$P(11)=\left\{\begin{array}{l}11^{\left(1^{(0,2,3,4,4,5,10,12,13)}, 2^{(0,1,3,3,6,7,10,12,13)}, 3^{(0,1,1,2,8,9,10,12,13)},\right)}, \\ 11^{\left(5^{(0,1,4,6,7,7,8,9,13)}, 6^{(0,2,4,4,7,7,8,9,10)}, 8^{(0,3,4,4,5,6,7,9,12)},\right)}, \\ 11^{\left(10^{(1,1,2,3,4,4,6,9,12,13)}, 12^{(1,2,2,3,4,7,7,8,10,13)}, 13^{(1,2,3,3,5,7,9,10,112)}\right)} .\end{array}\right.$

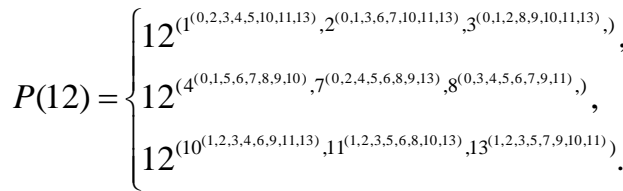

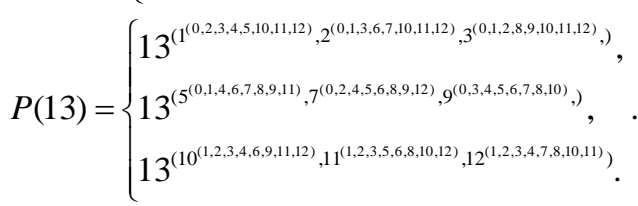

2. Из множества проекций $\left\{P\left(v_{j}\right)\right\}$ получим $\left\{P^{\prime}\left(v_{j}\right)\right\}$ :

$P^{\prime}(0)=\left\{\begin{array}{l}0^{\left(1^{(2,3,4,5)}, 2^{(1,3,6,7)}, 3^{(1,2,2,9)},\right)}, \\ 0^{\left(4^{(1,5,6,6,7,8,9)}, 5^{(1,4,6,6,7,8,9)}, 6^{(2,4,5,7,8,9)},\right)} \\ 0^{\left(7^{(2,4,4,5,6,8,9)}, 8^{(3,4,5,6,7,9)}, 9^{(3,4,5,6,7,8)}\right)} .\end{array}\right.$ 


\begin{tabular}{|c|c|c|c|c|c|c|}
\hline \multirow{4}{*}{ Impact Factor: } & ISRA (India) & $=3.117$ & SIS (USA) & $=0.912$ & ICV (Poland) & $=6.630$ \\
\hline & ISI (Dubai, UAE & $=0.829$ & РИНЦ (Russia) & $=0.156$ & PIF (India) & $=1.940$ \\
\hline & GIF (Australia) & $=0.564$ & ESJI (KZ) & $=\mathbf{5 . 0 1 5}$ & IBI (India) & $=4.260$ \\
\hline & JIF & $=1.500$ & SJIF (Morocco) & $=5.667$ & & \\
\hline
\end{tabular}

$$
\begin{aligned}
& P^{\prime}(1)=\left\{\begin{array}{l}
1^{\left(0^{(2,3,4,5)}, 2^{(0,3,10,11,12,13)}, 3^{(0,2,10,11,12,13)},\right)} \\
1^{\left(4^{(0 ., 5,10,12)}, 5^{(0,4,11,13)}, 10^{(2,3,4,4,11,12,13)},\right)}, \\
1^{\left(11^{(2,3,3,5,10,12,13)}, 12^{(2,3,4,4,10,11,13)}, 1^{(2,3,5,10,11,12)}\right)} .
\end{array}\right.
\end{aligned}
$$

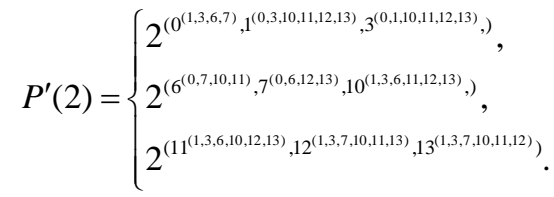

$$
\begin{aligned}
& P^{\prime}(3)=\left\{\begin{array}{l}
3^{\left(0^{(1,2,8,9)}, 1^{(0,2,1,1,11,12,13)}, 2^{(0,1,11,11,1,1,13)},\right)}, \\
3^{\left(8^{(0,9,11,112)}, 9^{(0,8,10,13)}, 10^{(1,2,9,11,11,13)},\right)}, \\
3^{\left(11^{(1,2,2,110,12,13)}, 12^{(1,2,8,10,11,113)}, 13^{(1,2,9,10,11,12)}\right)} .
\end{array}\right. \\
& P^{\prime}(4)=\left\{\begin{array}{l}
4^{\left(0^{(1,5,5,6,7,8,9)}, 1^{(0.5,10,12)}, 5^{(0.1,1,6,7,8,9)},\right)}, \\
4^{\left(6^{(0,5,7,7,8,910)}, 7^{(0.5,5,6,8,9,12)}, 8^{(0.5,6,6,7,9,12)},\right)}, \\
4^{\left(9^{(0.5,6,67,7,10)}, 10^{(1,6,9,12)}, 12^{(1,7,8,10)}\right)} .
\end{array}\right.
\end{aligned}
$$

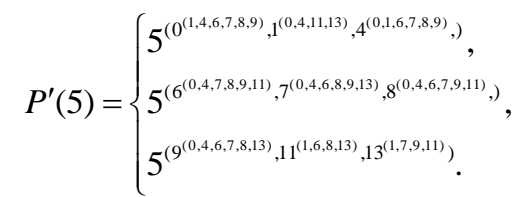

$$
\begin{aligned}
& P^{\prime}(6)=\left\{\begin{array}{l}
6^{\left(0^{(2,4,5,7,7,9)}, 2^{(0,7,10,11)}, 4^{(0,5,7,7,9,10)},\right)}, \\
6^{\left(5^{(0,4,4,7,9,911)}, 7^{(0,2,4,5,5,9)}, 8^{(0,4,5,7,9,11)},\right.}, \\
6^{\left(9^{(0,4,4,7,7,8,10)}, 10^{(2,4,4,11)}, 11^{(2,5,8,10)}\right)} .
\end{array}\right.
\end{aligned}
$$

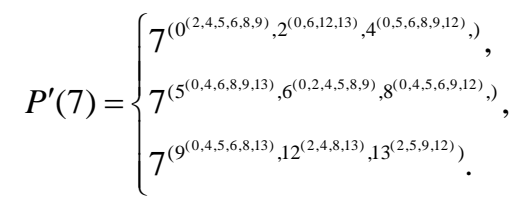

$$
\begin{aligned}
& P^{\prime}(8)=\left\{\begin{array}{l}
8^{\left(0^{(3,4,5,6,6,7,9)}, 3^{(0,9,11,12)}, 4^{(0,5,6,7,9,12)},\right)}, \\
8^{\left(5^{(0,4,6,6,7,9,1)}, 6^{(0,4,5,5,7,11)}, 7^{(0,4,5,6,6,12)},\right)} \\
8^{\left(9^{(0,3,4,5,5,6,7)}, 11^{(3,5,6,6,12)}, 12^{(3,4,7,711)}\right)} .
\end{array}\right. \\
& P^{\prime}(9)=\left\{\begin{array}{l}
9^{\left(0^{(3,4,5,6,6,7,8)}, 3^{(0,8,10,13)}, 4^{(0.5,6,6,7,8,10)},\right)}, \\
9^{\left(5^{(0,4,4,6,7,13)}, 6^{(0,4,5,7,7,8,10)}, 7^{(0,4,5,6,6,8,13)},\right)}, \\
9^{\left(8^{(0,3,3,4,5,6,7)}, 10^{(3,4,6,6,13)}, 13^{(3,5,5,7,10)}\right)} .
\end{array}\right. \\
& P^{\prime}(10)=\left\{\begin{array}{l}
10^{\left(1^{(2,3,4,11,12,13)}, 2^{(1,3,6,111,12,13)}, 3^{(1,2,9,11,11,13)},\right)}, \\
10^{\left(4^{(1,6,6,912)}, 6^{(2,4,9,11)}, 9^{(3,4,6,613)},\right)}, \\
10^{\left(11^{(1,2,3,3,6,12,13)}, 12^{(1,2,3,4,4,11,13)}, 13^{(1,2,3,9,11,12)}\right)} .
\end{array}\right. \\
& P^{\prime}(11)=\left\{\begin{array}{l}
11^{\left(1^{(2,3,5,10,12,13)}, 2^{(1,3,6,10,12,13)}, 3^{(1,2,8,10,12,13)},\right)}, \\
11^{\left(5^{(1,6,8,13)}, 6^{(2,5,5,10)}, 8^{(3,5,6,12)},\right)}, \\
11^{\left(10^{(1,2,2,3,6,12,13)}, 12^{(1,2,3,3,8,10,13)}, 13^{(1,2,3,5,10,12)}\right)} .
\end{array}\right.
\end{aligned}
$$

$$
\begin{aligned}
& P^{\prime}(12)=\left\{\begin{array}{l}
12^{\left(1^{(2,3,4,1,10,11,13)}, 2^{(1,3,7,10,11,13)}, 3^{(1,2,8,10,11,13)},\right)}, \\
12^{\left(4^{(1,7,7,10)}, 7^{(2,4,8,8,13)}, 8^{(3,4,7,11)},\right)}, \\
12^{\left(10^{(1,2,2,3,4,11,13)}, 11^{(1,2,2,3,8,10,13)}, 13^{(1,2,3,3,7,10,11)}\right)} .
\end{array}\right. \\
& P^{\prime}(13)=\left\{\begin{array}{l}
13^{\left(1^{(2,3,5,10,11,12)}, 2^{(1,3,7,10,11,12)}, 3^{(1,2,9,10,11,12)},\right.}, \\
13^{\left(5^{(1,7,9,11)}, 7^{(2,5,9,12)}, 9^{(3,5,7,10)},\right.}, \\
13^{\left(10^{(1,2,2,3,1,11,12)}, 1^{(1,2,3,3,5,10,12)}, 12^{(1,2,2,3,7,10,11)}\right)} .
\end{array}\right. \\
& \text { 3. } m:=\max m\left(P^{\prime}\left(v_{j}\right)\right)=6 \text {. }
\end{aligned}
$$

4.

$$
\begin{aligned}
& P^{\prime \prime}(0)=\left\{\begin{array}{l}
0^{\left(4^{(5,6,7,8,9)}, 5^{(4,6,7,8,9)}, 6^{(4,5,7,7,9)},\right)}, \\
0^{\left(7^{(4,5,6,8,9)}, 8^{(4,5,6,6,7,9)}, 9^{(4,5,6,7,7)}\right)} .
\end{array}\right. \\
& P^{\prime \prime}(1)=\left\{\begin{array}{l}
1^{\left(2^{(3,10,11,12,13)}, 3^{(2,10,11,11,13)}, 10^{(2,3,31,11,12,13)}\right)}, \\
1^{\left(11^{(2,3,10,12,13)}, 12^{(2,3,10,11,13)}, 13^{(2,3,1,10,11,12)}\right)} .
\end{array}\right. \\
& P^{\prime \prime}(2)=\left\{\begin{array}{l}
2^{\left(1^{(3,10,11,12,13)}, 3^{(1,10,11,12,13)}, 10^{(1,3,11,1,12,13)},\right)}, \\
2^{\left(11^{(1,3,3,10,12,13)}, 12^{(1,3,10,11,13)}, 13^{(1,3,10,11,12)}\right)} .
\end{array}\right. \\
& P^{\prime \prime}(3)=\left\{\begin{array}{l}
3^{\left(1^{(2,10,11,1,13)}, 2^{(1,1,1,11,12,13)}, 10^{(1,2,211,11,13)}\right)}, \\
3^{\left(11^{(1,2,1,1,12,13)}, 12^{(1,2,10,11,13)}, 13^{(1,2,10,11,12)}\right)} .
\end{array}\right. \\
& P^{\prime \prime}(4)=\left\{\begin{array}{l}
4^{\left(0^{(5,6,67,7,9)}, 5^{(0,6,7,7,9)}, 6^{(0,5,7,8,9)},\right)}, \\
4^{\left(7^{(0,5,5,6,8,9)}, 8^{(0 ., 5,6,7,9)}, 9^{(0 ., 5,6,7,8)}\right)} .
\end{array}\right. \\
& P^{\prime \prime}(5)=\left\{\begin{array}{l}
5^{\left(0^{(4,6,7,8,9)}, 4^{(0,6,7,7,9)}, 6^{(0,4,7,8,9)},\right)}, \\
5^{\left(7^{(0,4,6,6,8)}, 8^{(0,4,6,7,9)}, 9^{(0.4,6,7,7)}\right)} .
\end{array},\right. \\
& P^{\prime \prime}(6)=\left\{\begin{array}{l}
6^{\left(0^{(4,5,7,8,9)}, 4^{(0.5,7,7,8)}, 5^{(0,4,7,7,9)},\right)}, \\
6^{\left(7^{(0,4,5,8,9)}, 8^{(0,4,5,5,79)}, 9^{(0,4,5,5,7)}\right)} .
\end{array}\right. \\
& P^{\prime \prime}(7)=\left\{\begin{array}{l}
7^{\left(0^{(4,5,6,6,9)}, 4^{(0.5,6,8,9)}, 5^{(0,4,6,8,9)},\right)}, \\
7^{\left(6^{(0,4,5,5,9)}, 8^{(0,4,5,6,9)}, 9^{(0,4,5,6,8)}\right)} .
\end{array}\right. \\
& P^{\prime \prime}(8)=\left\{\begin{array}{l}
8^{\left(0^{(4,5,6,7,9)}, 4^{(0,5,6,7,9)}, 5^{(0,4,6,7,9)},\right)}, \\
8^{\left(6^{(0,4,5,7,9)}, 7^{(0,4,5,6,9)}, 9^{(0,4,5,6,7)}\right)} .
\end{array}\right. \\
& P^{\prime \prime}(9)=\left\{\begin{array}{l}
9^{\left(0^{(4,5,6,6,7)}, 4^{(0.5,6,7,7)}, 5^{(0,4,6,7,8)},\right.}, \\
9^{\left(6^{(0,4,4,7,8)}, 7^{(0,4,5,6,8)}, 8^{(0,4,5,6,7)}\right)} .
\end{array}\right. \\
& P^{\prime \prime}(10)=\left\{\begin{array}{l}
10^{\left(1^{(2,3,11,11,13)}, 2^{(1,3,1,1,1,13)}, 3^{(1,2,21,11,13)}\right)}, \\
10^{\left(11^{(1,2,3,12,13)}, 1^{(1,2,3,11,13)}, 1^{(1,2,3,11,12)}\right)} .
\end{array}\right. \\
& P^{\prime \prime}(11)=\left\{\begin{array}{l}
11^{\left(1^{(2,3,10,12,13)}, 2^{(1,3,10,11,13)}, 3^{(1,2,10,11,13)},\right)}, \\
11^{\left(10^{(1,2,3,12,13)}, 12^{(1,2,3,10,13)}, 1^{(1,2,3,10,12)}\right)} .
\end{array}\right. \\
& P^{\prime \prime}(12)=\left\{\begin{array}{l}
12^{\left(1^{(2,3,1,10,11,13)}, 2^{(1,3,110,11,13)}, 3^{(1,2,10,11,113)},\right)}, \\
12^{\left(10^{(1,2,3,11,13)}, 11^{(1,2,3,10,13)}, 1^{(1,1,2,3,10,11)}\right)} .
\end{array}\right. \\
& P^{\prime \prime}(13)=\left\{\begin{array}{l}
13^{\left(1^{(2,3,10,11,12)}, 2^{(1,3,10,11,12)}, 3^{(1,2,10,11,12)}\right)}, \\
13^{\left(1^{(1,1,3,11,12)}, 11^{(1,2,3,10,12)}, 12^{(1,2,3,10,11)}\right)} .
\end{array}\right.
\end{aligned}
$$

Получили $m=5, n_{1}=6,\left|K_{\max }(G)\right|=7$. 


\begin{tabular}{|c|c|c|c|c|c|c|}
\hline \multirow{4}{*}{ Impact Factor: } & ISRA (India) & $=3.117$ & SIS (USA) & $=0.912$ & ICV (Poland) & $=6.630$ \\
\hline & ISI (Dubai, UAE & $=0.829$ & РИНЦ (Russia) & $=0.156$ & PIF (India) & $=1.940$ \\
\hline & GIF (Australia) & $=0.564$ & ESJI (KZ) & $=\mathbf{5 . 0 1 5}$ & IBI (India) & $=4.260$ \\
\hline & JIF & $=1.500$ & SJIF (Morocco) & $=5.667$ & & \\
\hline
\end{tabular}

$V(0)=V(4)=V(5)=V(6)=V(7)=V(8)=V(9)$ $=(0,4,5,6,7,8,9)=K_{1}(G)$.

$V(1)=V(2)=V(3)=V(10)=V(11)=V(12)=$ $V(13)=(1,2,3,10,11,12,13)=K_{2}(G)$.

Как видим, каждая из полученных проекций описывает 7-клику, и клик таких две: $(0,4,5,6,7,8,9)$ и $(1,2,3,10,11,12,13)$. Исходя из этого, определим масштабируемость рассмотренного здесь графа 2-достижимости:

$$
\mu_{2}(G)=\varphi_{2}(G) / n(G)=7 / 14=0,5 .
$$

Так как диаметр графа 2 (рис. 2) равен трем, то граф 3-достижимости полносвязен, поэтому

$$
\varphi_{3}(G) / n(G) \text { и } \mu_{3}(G)=1 \text {. }
$$

Топологическая отказоустойчивость

Для исследования топологических аспектов устойчивости ВС к отказам кратности $l$ в данной работе мы используем графы $\partial(k)$-достижимости, в которых смежность вершин, в отличие от графов д-достижимости, лимитирована не только допускаемым расстоянием $\partial$ между ними, но и числом $k=l+1$ независимых путей с длиной, не превышающей этого расстояния [14, с. 239]. В этом смысле в работе [17, с. 101] Автором приведено не вполне адекватное определение графа $\partial(k)$-достижимости $G_{\partial(k)}(G)$, как надграфа графа $G(V, E)$. На самом деле граф $\partial(k)$ достижимости $G_{\partial(k)}(G)$ является подграфом графа $\partial$-достижимости $G_{\partial}(G)$, в котором удалены ребра между вершинами $u, v, \in V$, если в исходном графе $G$ существует менее $k$ независимых путей с длиной, не превышающей значение заданной достижимости $\partial$. Понятно, что число $k$ таких путей (соединимость информационно смежных в подсистеме вершин подграфа системы) должно по меньшей мере превышать заданную кратность $l$ отказов: $k \geq l+1$. Тогда при удалении $l$ какихлибо вершин из соответствующего выполняемой задаче подграфа ВС любые две из оставшихся в нем информационно смежных вершин гарантированно останутся соединенными путем с длиной, не превышающей предельной для этой задачи достижимости $\partial$. О выполнении условия смежности вершин $u, v, \in V$ в графе $G_{\partial(k)}(G)$ можно судить по наличию не менее $k$ реплик вершин $u$ или $v$ на ? уровнях соответствующих этим вершинам проекций $P(v)$ или $P(u)$ исходного графа $G$.

В данной работе мы ограничимся построением 2-уровневых проекций графа $\partial(k)$-достижимости $G_{\partial(k)}(G) \quad$ на примере исходного графа 2, приведенного вместе с его полными проекциями на рис. 2. Этого достаточно для выявления в таком графе наибольшей устойчивой к однократным отказам клики $(l=$ $1, k=2)$.

Как видим, и на первом и на втором уровнях исходных проекций реплики каких-либо вершин отсутствуют, следовательно, графы $G_{\partial(2)}(G)$ и при $\partial=1$, и при $\partial=2$ представлены множествами изолированных вершин, поэтому исследование их устойчивости к отказам не имеет смысла. В связи с этим далее мы продемонстрируем исследование устойчивости заданной графом 2 топологии ВС к однократным $(l=1, k=2)$ отказам и при достижимости $\partial=3$.

Построение проекции графа $G_{3(2)}(G)$ покажем на примере проекции $P(0)$ :

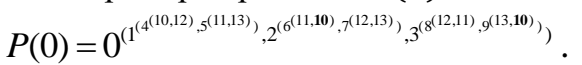

Как видим, оригинал вершины 10 имеет две реплики (выделены жирным шрифтом), что даже превышает первоначально заданное значение допускаемой кратности $l=1$. Отметим также, что все 3 пути из ракурсной вершины в вершину 10 и в ее реплики не имеют пересечений и, таким образом, являются независимыми: $(0,1,4,10)$, $(0,2,6,10)$ и $(0,3,9,10)$. То же самое можно отнести к вершинам 11, 12, 13 проекции $P(0)$. Тогда 1уровневая проекция $P_{3(2)}(0)$ имеет вид:

$$
P_{3(2)}(0)=0^{(10,11,12,13)} \text {. }
$$

Получив таким же образом остальные 1-уровневые проекции графа 3(2)-достижимости:

$$
\begin{array}{ll}
P_{3(2)}(0)=0^{(10,11,12,13)}, & P_{3(2)}(1)=1^{(6,7,8,9),} \\
P_{3(2)}(2)=2^{(4,5,8,9)}, & P_{3(2)}(3)=3^{(4,5,6,7)}, \\
P_{3(2)}(4)=4^{(2,3,11,13)}, & P_{3(2)}(5)=5^{(2,3,10,12)}, \\
P_{3(2)}(6)=6^{(1,3,12,13)}, & P_{3(2)}(7)=7^{(1,3,10,11)}, \\
P_{3(2)}(8)=8^{(1,2,10,13)}, & P_{3(2)}(9)=9^{(1,2,11,12)}, \\
P_{3(2)}(10)=10^{(0,5,7,8)}, & P_{3(2)}(11)=11^{(0,4,7,9)}, \\
P_{3(2)}(12)=12^{(0,5,6,9)}, & P_{3(2)}(13)=13^{(0,4,6,8)},
\end{array}
$$

преобразуем их в 2-уровневые, заменив вершины 1-го уровня их 1-уровневыми проекциями:

1.

$$
\begin{aligned}
& P_{3(2)}(0)=0^{\left(10^{(5,7,8)}, 11^{(4,7,9)}, 12^{(5,6,9)}, 13^{(4,6,8)}\right)}, \\
& P_{3(2)}(1)=1^{\left(6^{(3,12,13)}, 7^{(3,10,11)}, 8^{(2,10,13)}, 9^{(2,11,12)}\right)} \text {, } \\
& P_{3(2)}(2)=2^{\left(4^{(3,11,13)}, 5^{(3,10,12)}, 8^{(1,10,13)}, 9^{(1,11,12)}\right)} \text {, } \\
& P_{3(2)}(3)=3^{\left(4^{(2,11,13)}, 5^{(2,10,12)}, 6^{(1,12,13)}, 7^{(1,10,11)}\right)} \text {, } \\
& P_{3(2)}(4)=4^{\left(2^{(5.8 .9)}, 3^{(5,6,7)}, 11^{(0.7 .9)}, 13^{(0,6,8)}\right)} \text {, } \\
& P_{3(2)}(5)=5^{\left(2^{(4,8,9)}, 3^{(4,6,7)}, 10^{(0,7,8)}, 12^{(0,6,9)}\right)} \text {, } \\
& P_{3(2)}(6)=6^{\left(1^{(7.8,9)}, 3^{(4,5,7)}, 12^{(0,5,9)}, 13^{(0.4,8)}\right)}, \\
& P_{3(2)}(7)=7^{\left(1^{(6,8,9)}, 3^{(4.5,5)}, 10^{(0.5,8)}, 11^{(0,4,9)}\right)} \text {, } \\
& P_{3(2)}(8)=8^{\left(1^{(6,7,9)}, 2^{(4,5,9)}, 10^{(0,5,7)}, 13^{(0,4,6)}\right)} \text {, } \\
& P_{3(2)}(9)=9^{\left(1^{(6,7.8)}, 2^{(4.5,8)}, 11^{(0.4,7)}, 12^{(0.5,6)}\right)}, \\
& P_{3(2)}(10)=10^{\left(0^{(11,12,13)}, 5^{(2,3,12)}, 7^{(1,3,31)}, 8^{(1,2,13)}\right)} \text {, } \\
& P_{3(2)}(11)=11^{\left(0^{(10,12,13)}, 4^{(2,3,13)}, 7^{(1,3,10)}, 9^{(1,2,12)}\right)} \text {, } \\
& P_{3(2)}(12)=12^{\left(0^{(10,11,13)}, 5^{(2,3,10)}, 6^{(1,3,13)}, 9^{(1,2,11)}\right)} \text {, }
\end{aligned}
$$




\begin{tabular}{|c|c|c|c|c|c|c|}
\hline \multirow{4}{*}{ Impact Factor: } & ISRA (India) & $=3.117$ & SIS (USA) & $=0.912$ & ICV (Poland) & $=6.630$ \\
\hline & ISI (Dubai, UAE & $=0.829$ & РИНЦ (Russia) & $=0.156$ & PIF (India) & $=1.940$ \\
\hline & GIF (Australia) & $=0.564$ & ESJI (KZ) & $=\mathbf{5 . 0 1 5}$ & IBI (India) & $=4.260$ \\
\hline & JIF & $=1.500$ & SJIF (Morocco) & $=5.667$ & & \\
\hline
\end{tabular}

$$
P_{3(2)}(13)=13^{\left(0^{(10.11,12)}, 4^{(2,3,11)}, 6^{(1,3,12)}, 8^{(1,2,10)}\right)} \text {. }
$$

2. Получим пустые подмножества вторых уровней каждой из проекций.

3. Получим $m:=0$.

Таким образом порядок наибольшей клики равен двум, и любая пара смежных в графе 3(2)достижимости $G_{3(2)}(G)$ вершин является наибольшей в нем кликой порядка $2, \varphi_{3(2)}(G)=2$. Напомним, что речь здесь идет о смежности вершин в графе $G_{3(2)}(G)$, а не в исходном графе $G$. Тогда топологическая масштабируемость

$$
\mu_{3(2)}(G)=\varphi_{3(2)}(G) / n(G)=2 / 14=0,143 .
$$

Топологическая $l$-отказоустойчивость $\theta_{n, \partial}(l)$ системы с $G$-топологией $(n \equiv n(G))$ в решении $\partial$ задач (задач, допускающих достижимость ?) определена в $[18$, с. 78$]$ отношением плотности $\varphi_{\partial(k)}(G) \equiv \varphi\left(G_{\partial(k)}\right)$ графа $\partial(k)$-достижимости $G_{\partial(k)}$ к исключающей наличие отказов $(l=0)$ плотности $\varphi_{\partial}(G) \equiv \varphi\left(G_{\partial}\right)$ графа $\partial$-достижимости $G_{\partial}$ :

$$
\theta_{l, \partial}(G)=\varphi_{\partial(k)}(G) / \varphi_{\partial}(G),
$$

или, что равносильно, отношением соответствующих значений масштабируемости:

$$
\theta_{l, \partial}(G)=\mu\left(G_{\partial(k)}\right) / \mu\left(G_{\partial}\right) .
$$

Учитывая полученные в предыдущем разделе значения плотности $\varphi_{3}(G)=14$ и масштабируемости $\mu_{3}(G)=1$, а также найденные здесь значения плотности $\varphi_{3(2)}(G)=2$ и масштабируемости $\quad \mu_{3(2)}(G)=0,143$, топологическая устойчивость $\theta_{1,3}(G)$ к однократным отказам заданной графом $G$ рассматриваемой $\mathrm{BC}$ при решении на ней задач, в которых расстояния между информационносмежными ветвями не превышают $\partial=3$, составит:

$$
\theta_{1,3}(G)=2 / 14=0,143 / 1=0,143 .
$$

Далее мы покажем, каким образом изменится топологическая отказоустойчивость этой же системы при решении на ней задач, допускающих предельные расстояния между информационносмежными ветвями $\partial=4$. Приведем полученные из исходных проекций графа 2 (рис. 2) одноуровневые проекции $P_{4(2)}\left(v_{j}\right), v_{j} \in V$ :

$$
\begin{aligned}
& P_{4(2)}(0)=0^{(4,5,6,7,7,9,10,11,1,13,13)}, \quad P_{4(2)}(1)=1^{(2,3,6,7,7,9,9,10,11,12,13)}, \\
& P_{4(2)}(2)=2^{(1,3,4,5,8,9,9,10,11,1,1,13)}, \quad P_{4(2)}(3)=3^{(1,2,4,5,6,6,7,10,11,1,1,13)} \text {, } \\
& P_{4(2)}(4)=4^{(0,2,3,5,6,7,7,9,9,1,13)}, \quad P_{4(2)}(5)=5^{(0,2,3,4,6,6,7,9,9,10,12)}, \\
& P_{4(2)}(6)=6^{(0,1,3,4,5,7,8,9,1,12,13)}, \quad P_{4(2)}(7)=7^{(0,1,3,4,5,6,8,9,10,11)}, \\
& P_{4(2)}(8)=8^{(0,1,2,4,5,6,6,7,9,10,13)}, \quad P_{4(2)}(9)=9^{(0,0,1,2,4,5,6,7,7,8,11,12)} \text {, } \\
& P_{4(2)}(10)=10^{(0,1,2,3,5,7,7,8,11,12,13)}, P_{4(2)}(11)=11^{(0,1,2,3,4,7,7,1,10,12,13)} \text {, } \\
& P_{4(2)}(12)=12^{(0,1,2,3,5,5,6,9,10,11,13)}, P_{4(2)}(13)=13^{(0,1,2,3,4,6,6,8,10,11,12)} \text {. }
\end{aligned}
$$

Не будем здесь приводить промежуточных действий, укажем лишь результат применения алгоритма: граф $G_{3(2)}$ (граф 3-достижимости и 2соединимости) имеет две непересекающиеся 7- клики - $\quad(0,4,5,6,7,8,9)$ и $\quad(1,2,3,10,11,12,13)$. Топологическая масштабируемость такой ВС при допускаемой достижимости $\mu_{4(2)}(G) \equiv \mu\left(G_{4(2)}\right)=$ $7 / 14=0,5$.

Топологическую $l$-отказоустойчивость $\theta_{n, \partial}(l)$ системы с $G$-топологией $(n \equiv n(G))$ в решении информационно-полносвязных $\partial$-задач (задач, допускающих расстояния между процессорами подсистемы, реализующих параллельные ветви, не превышающие значения $\partial$ ) определим отношением плотности $\varphi_{\partial(k)}(G) \equiv \varphi\left(G_{\partial(k)}\right)$ графа $\partial(k)$-достижимости $\quad G_{\partial(k)},(k=l+1) \quad$ к исключающей наличие отказов $(l=0, k=1)$ плотности $\varphi_{\partial}(G) \equiv \varphi\left(G_{\partial}\right)$ графа $\partial$-достижимости $G_{\partial}:$

$$
\theta_{\partial(l+1)}^{l}(G)=\mu\left(G_{\partial(l+1)}\right) / \mu\left(G_{\partial}\right)=\varphi\left(G_{\partial(l+1)}\right) / \varphi\left(G_{\partial}\right) .
$$

Итак, выше мы показали, что свойство устойчивости к однократным отказам $(l=1, k=$ $2)$ в системе с рассматриваемой здесь топологией (граф 2, рис. 2) возникает только для задач, допускающих достижимость параллельных ветвей не ниже четырех, при этом $\mu_{4(2)}(G)=0,5$. В вышерасположенном разделе показано, что $\varphi\left(G_{\partial \geq 3}\right)$ кликовое число (плотность) графа рассматриваемой системы при достижимости $\partial \geq$ 3 равно порядку $n$ этого графа $\left(\varphi\left(G_{\partial \geq 3}\right)=14\right.$. Таким образом, топологическая отказоустойчивость такой системы -

$$
\theta_{4(2)}^{1}(G)=\varphi\left(G_{4(2)}\right) / \varphi\left(G_{4}\right)=0,5 / 1,0=0,5
$$

\section{Заключение}

Проблема сопоставления топологий в по обеспечиваемому топологией потенциалу параллелизма вычислительной системы связана с выявлением наибольших клик в ее графе. Предлагаемое в работе решение этой задачи основано на представлении графа его двухуровневыми проекциями, определены свойства проекций полного графа, позволяющие выделить максимальный полный подграф в произвольном графе. Даны вербальное и формальное описание соответствующего алгоритма, на примерах показано его применение.

Даны понятия показателей топологической масштабируемости и топологической отказоустойчивости вычислительных систем. На примерах показаны: преобразование проекций исходного графа ВС в 2-уровневые проекции графов с предельно допускаемыми в ней значениями достижимости и кратности отказов, выявление в этих графах наибольших клик и топологический анализ системы с заданными значениями достижимости и кратности отказов.

Концептуальность понятия клик в теории графов общеизвестна, поэтому Автор уверен, что предложенное в работе решение задачи поиска 


\begin{tabular}{|c|c|c|c|c|c|c|}
\hline \multirow{4}{*}{ Impact Factor: } & ISRA (India) & $=3.117$ & SIS (USA) & $=0.912$ & ICV (Poland) & $=6.630$ \\
\hline & ISI (Dubai, UAE & $=0.829$ & РИНЦ (Russia) & $=0.156$ & PIF (India) & $=1.940$ \\
\hline & GIF (Australia) & $=0.564$ & ESJI (KZ) & $=\mathbf{5 . 0 1 5}$ & IBI (India) & $=4.260$ \\
\hline & JIF & $=1.500$ & SJIF (Morocco) & $=5.667$ & & \\
\hline
\end{tabular}

наибольших клик в графе ВС будет востребовано не только в теории и практике вычислительных систем, но, прежде всего, непосредственно в теории графов, а также в других, базирующихся на методах этой теории отраслях науки и техники.

\section{References:}

1. Newman, M. E. J. (2005). A measure of betweenness centrality based on random walks. Social Networks, V. 27, 39-54. https://arxiv.org/pdf/cond-mat/0309045v1.pdf

2. Donetti, L., Hurtado, P. I., \& Munoz, M. A., (2005). Entangled Networks, Synchronization, and Optimal Network Topology. Phys. Rev. Lett. $\quad$ 95, 188701, 1-4. http://ic1.ugr.es/members/phurtado/wpcontent/uploads/sites/3/2016/11/PRL-95188701-2005.pdf

3. Melent'ev, V. A. (2015). On topological scalability of computing systems. UBS, 58, $115-143$.

http://ubs.mtas.ru/archive/search results_new.p hp?publication id=20724

4. Badekha, I., \& Roldugin, P. (2014). Density of graphs in which each edge is contained in at least two maximal cliques. Discrete Mathematics and Applications, 24(1), 1-12. https://www.degruyter.com/view/j/dma.2014.24 issue-1/dma-2014-0001/dma-2014-0001.xml

5. Christofides, N. (1975). Graph Theory: An algorithmic Approach. New York: Academic Press.

6. Kornushko, V., Bogunova, I., Panov, A., Nikolaeva, O., \& Flid, A. (2018). The application of the system approach for building the information space for the development of the production of ready medicines. Journal of Applied Informatics, V. 13, Issue 3(75), 83-100. https://elibrary.ru/item.asp?id=35224068

7. Volkova, A. (2018). A Technical Translation of Melentiev's Graph Representation Method with Commentary. (p.503). University Honors Theses.

https://pdxscholar.library.pdx.edu/honorstheses/ $\underline{503}$

8. Melentiev, V. A. (2000). Bracket Form of Graph Description and its Use in Structural Investigation of Robust Computer Systems. Optoelectronics, Instrumentation and Data Processing, No 4, Allerton Press Inc., USA, 3447. http://elibrary.ru/item.asp?id=14954075
9. Melent'ev, V. A. (2004). The formal rudiments of a bracket patterns' in graph theory. Proceedings of the II International Conference "Parallel Computations and Control Problems" PACO '2004. (pp.694-706). Moscow, 4-6 October 2004.

10. Melent'ev, V. A. (2005). Formal'nyj podhod k issledovaniyu struktur vychislitel'nyh sistem. Vestnik Tomskogo gosudarstvennogo universiteta, 14, 167-172.

11. Melent'ev, V. A. (2010). An analytical approach to the synthesis of regular graphs with preset values of the order, degree and girth. Prikl. Diskr. Mat., no. 2(8), 74-86. http://mi.mathnet.ru/eng/pdm178

12. Melent'ev, V. A. (2011). Compact structures of computer systems and their synthesis. UBS, 32, 241-261. http://mi.mathnet.ru/eng/ubs536

13. Melent'ev, V. A. (2012). The Metric, Cyclomatic and Synthesis of Topology of Systems and Networks. Proceedings of the 6th International Conference, "Parallel Computations and Control Problems" PACO '2012. (pp.10-25). Moscow, 24-26 Oct. 2012. V 3.

14. Melent'ev, V. A. (2014). Embedding of subsystems limiting length and number of paths between vertexes of computing system graph. UBS, 47, 212-246. http://mi.mathnet.ru/eng/ubs749

15. Melent'ev, V. A., Shubin, V. I., \& Zadorozhny, A. F. (2015) Topological Scalability of hypercubic parallel Systems and Tasks. ISJ Theoretical \& Applied Science, 11 (31), 122129.

http://dx.doi.org/10.15863/TAS.2015.11.31.19

16. Zykov, A. A. (1987). Foundations of graph theory. (p.384). Moskva: Nauka.

17. Melent'ev, V. A. (2016). Fault-tolerance of hypercubic and compact topology of computing systems. ISJ Theoretical \& Applied Science, 12 (44), 98-105. http://dx.doi.org/10.15863/TAS.2016.12.44.20 


\begin{tabular}{|c|c|c|c|c|c|c|}
\hline \multirow{4}{*}{ Impact Factor: } & ISRA (India) & $=3.117$ & SIS (USA) & $=0.912$ & ICV (Poland) & $=6.630$ \\
\hline & ISI (Dubai, UAE & $=0.829$ & РИНЦ (Russia) & $=0.156$ & PIF (India) & $=1.940$ \\
\hline & GIF (Australia) & $=0.564$ & ESJI (KZ) & $=\mathbf{5 . 0 1 5}$ & IBI (India) & $=4.260$ \\
\hline & JIF & $=1.500$ & SJIF (Morocco) & $=5.667$ & & \\
\hline
\end{tabular}

18. Melent'ev, V. A. (2017). On topological faulttolerance of scalable computing systems. UBS,

70, 58-86. http://mi.mathnet.ru/eng/ubs935 